\title{
The surgical management of hyperparathyroidism and endocrine disease of the pancreas in the multiple endocrine neoplasia type 1 patient
}

\author{
N. W. THOMPSON \\ Division of Endocrine Surgery. University of Michigan, Department of Surgery, Ann Arbor, Michigan. USA
}

\begin{abstract}
Thompson NW (Division of Endocrine Surgery, University of Michigan, Department of Surgery, Ann Arbor, Michigan, USA). The surgical management of hyperparathyroidism and endocrine disease of the pancreas in the multiple endocrine neoplasia type 1 patient (Minisymposium: Multiple Endocrine Neoplasia 1). J Intern Med 1995; 238: 269-80.
\end{abstract}

The surgical management of multiple endocrine neoplasia type 1 (MEN1) parathyroid disease and involvement of the endocrine pancreas remains controversial. Hyperparathyroidism, usually the first clinical manifestation of the syndrome, requires surgical treatment in nearly all patients. We favour a subtotal parathyroidectomy and cervical thymectomy rather than a total parathyroidectomy and autotransplant because of good long-term results and the absence of permanent hypoparathyroidism.
The results of treating 34 MEN1 patients during a 20-year period are reported. The most common functional pancreatic or duodenal tumours in MEN1 patients are gastrinomas and insulinomas. In addition to the management of functional syndromes, another major concern is the malignant potential of the neuroendocrine tumours that frequently develop. Our surgical management of gastrinomas and the ZES has evolved over a period of 15 years. We have found that distal pancreatectomy, enucleation of any neoplasms in the head, and duodenotomy and excision of any neuroendocrine tumours (gastrinomas) combined with a regional node dissection are effective in the majority of patients. The results of treating 21 MEN1 patients with ZES are reported.

Keywords: MEN1, hyperparathyroidism ZES, gastrinomas, neuroendocrine tumors.

\section{Introduction}

The surgical management of multiple endocrine neoplasia type 1 (MEN1) involvement in the parathyroid glands and pancreas remains controversial. Nearly all patients develop hyperparathyroidism (HPT), which is most frequently the first clinical manifestation of the syndrome [1-4]. Overt pancreatic disease is less frequent and usually develops at a later age than the HPT $[1,5]$. It may initially be detected after the development of specific symptoms caused by the hypersecretion of one or more of the gastrointestinal peptide hormones, mechanical effects of a nonfunctional tumour, or by screening imaging of the pancreas or liver. The most common functional pancreatic or duodenal tumours in MEN1 patients are gastrinomas and their management is the subject of the greatest controversy [1]. Their importance lies in the fact that up to $30 \%$ of MEN 1 patients will develop gastrinomas and the Zollinger- 
Ellison syndrome (ZES). The surgical management of MEN1 ZES has gradually evolved during a period of more than a decade in which important developments in pathology, localization techniques and drug therapy have played a significant role in determining the multifaceted operation we currently employ [6-18]. The optimal surgical management of insulinomas in MEN1 patients is less controversial, but as yet, not standardized $[19,20]$. The purpose of this paper is to report our experience at the University of Michigan. Ann Arbor, MI. USA, in the management of the MEN1 patient with either or both HPT, and neuroendocrine disease of the pancreas or duodenum.

\section{Hyperparathyroidism}

Multiglandular disease is present in all MEN1 patients with HPT $[1-3,8]$. Furthermore. it is assumed that up to $20 \%$ of patients will have a supernumerary or fifth gland within the thymus that must be considered in operative strategy $[1-3,21]$. Frequently, one or more parathyroid glands may be normal in size and even in microscopic appearance from a limited biopsy. The asymmetrical parathyroid enlargement by a nodular type of hyperplasia is typical of MEN1 [1]. Only one or two parathyroid glands may be enlarged grossly, and a false impression that the disease is caused by an adenoma or double adenoma' may occur, especially in the patient without a known family history or other manifestation of the MEN1 syndrome. Two surgical procedures are widely employed in treating MEN1 HPT [1, 4, 22-29]. Both are based on the assumption that persistent or recurrent hypercalcemia can be anticipated if less than a subtotal parathyroidectomy is performed. One technique is to perform a total parathyroidectomy with excision of the cervically accessible thymus gland and autotransplantation of sufficient parathyroid tissue into the forearm or other readily accessible location so that. should recurrence ever develop, re-operation using a local anaesthetic could be performed on an outpatient basis [27]. This procedure has the potentlal for permanent hypoparathyroidism if the transplanted tissue does not survive and the patients require replacement therapy with vitamin $D$ and oral calcium until the graft functions adequately (4-8 weeks or longer). Its advantage is that, if recurrence develops, a second procedure in the neck can usually be avoided, providing all parathyroid tissue has been excised at the first operation. Graft size reduction can be accomplished readily under local anaesthesia. The second procedure is a subtotal parathyroidectomy with cervical thymectomy and is the one that we have used during the past 20 years and currently prefer $[1,26]$. Our surgical explorations in MEN1 patients have included a careful search for a minimum of four parathyroid glands and excision of the thymus. All parathyroid glands but one are excised. When all four glands are enlarged, a well vascularized gland is partially resected leaving a remnant weighing approximately $50-60 \mathrm{mg}$. When a normalsized gland has been identified, regardless of its location, it is carefully preserved and marked with a metal clip for possible future identification. When all four glands are enlarged, the smallest inferior gland is mobilized and trimmed to the desired size after carefully placing a metal clip across the gland. The resected portion is cut on the clip after packing the surrounding area with gauze sponges to prevent possible implantation of hyperplastic parathyroid cells. The remnant with clip is then tacked with a stitch to the trachea for easy access should a subsequent recurrence caused by remnant hypertrophy develop.

\section{Results}

Thirty-four MEN1 patients with HPT treated at the University of Michigan Medical Center between 1972 and 1994 were available for follow-up studies ranging from 3 months to 20 years following parathyroidectomy.

Twenty-four patients had their primary operations at the University of Michigan. One of these patients. in whom only three parathyroid glands were found at exploration, had persistent hypercalcemia (4.1\%). At re-operation, the missing left inferior parathyrotd gland was found within the lower pole of the thyroid gland. Three patients developed recurrent hyperparathyroidism diagnosed at Intervals of 8,16 and 17 years after a subtotal parathyroidectomy and thymectomy (12.5\%). All three patients were reoperated and found to have remnant hypertrophy. Partial resection of the remnant has resulted in normocalcemia in each case. Twenty-three out of the 24 patients became transiently hypocalcemic after their initial operations and most required vitamin $D$ preparations (Dihydrotachysterol) in addition to oral 
calcium carbonate supplements. Similarly, transient hypocalcemia developed in the four patients who were subsequently re-explored. No patient has developed permanent hypocalcemia, although one is taking daily oral calcium supplements.

A subset of this group of 24 patients consists of seven patients who had synchronous primary HPT, and neuroendocrine pancreatic or duodenal neuroendocrine tumours. Each underwent a combined cervical and abdominal exploration, avoiding a second operative procedure. These patients, all explored between 1990 and 1993, are currently normocalcemic following subtotal parathyroidecomy and cervical thymectomy. Each of these patients llso underwent a distal pancreatectomy. Five subjects with ZES also had duodenotomies and excision of submucosal gastrinomas. One patient with hypoglycemia had multiple neuroendocrine tumours in the neck, body and tail of the pancreas. One patient without symptoms was found to have a neuroendocrine tumour within the pancreatic head on routine CT screening. Based on the favourable results in this group, we recommend this combined approach for synchronous HPT and pancreatic disease, providing neither neck nor abdominal procedure is a re-operative exploration. None of these procedures exceeded $5 \mathrm{~h}$ in operative time.

Eleven patients who were initially treated elsewhere underwent re-operative parathyroidectomies for persistent $(n=8)$ or recurrent disease $(n=3)$. A subtotal parathyroidectomy was accomplished in eight patients in which one or more enlarged hyperplastic glands was excised. Each patient also had a cervical thymectomy. The three patients with recurrences were found to have hypertrophy of remnants which were trimmed to an estimated 50-60 mg. One out of the 11 patients has persistent hypercalcemia and another has serum calcium levels at the upper limit of normal. All but one patient was temporarily hypocalcemic, although none developed permanent hypoparathyroidism.

Thirty-four out of the 35 MEN1 patients are normocalcemic. The one patient with persistent hypercalcemia is asymptomatic. However, it should be emphasized that 15 out of these 34 patients required two operations (44\%), nine for persistent and six for recurrent disease. Remnant hypertrophy was the only cause of true recurrence in this group of patients and occurred at intervals ranging from 4 to 17 years after an initially successful operation. Re- operation for remnant recurrences was not difficult in those cases in which the surgeon had carefully documented the location of the remaining gland. Annual follow-up of these patients is indicated for life because late recurrences can be anticipated and occurred in $17.6 \%$ of all patients in this series. We favour subtotal parathyroidectomy and thymectomy because, if carefully performed, it completely avoids permanent hypoparathyroidism, and in our own primary cases, was associated with a long-term recurrence rate of only $12.5 \%$. Furthermore, reoperation in this group was successful without any significant morbidity.

\section{Surgical disease of the pancreas and duodenum}

During the past 2 decades, several important developments have contributed to a more rational approach to the operative management of neuroendocrine disease in MEN1 patients. The first was the finding on immunohistochemical studies of MEN 1 pancreas and tumours that patients with functional hormonal syndromes had one or more discreet tumours as the cause of the syndrome, rather than islet-cell hyperplasia or microadenomastosis, even though the diffuse changes always accompanied the tumour(s) $[7,8]$. As a result, it became apparent that functional syndromes might be effectively treated if all gross tumour(s) could be excised without the need for total pancreatectomy. Another finding was that most patients with ZES had small gastrinomas arising within the duodenal wall which could be the only cause of hypergastrinemia. In many of these cases. gross tumours elsewhere in the pancreas were present, but based on immunohistochemical studies. were not found to be the cause of the ZFS in the majority of patients $[6,8,10,18,30-33]$. Finally, it was discovered that duodenal gastrinomas, despite sizes as small as $1-2 \mathrm{~mm}$, metastasized to the peripancreatic and periduodenal lymph nodes and were accompanied by liver metastases in about only $10 \%$ of cases, and usually, only after a long period without surgical intervention $[9,13,14,16,17$, 21, 32, 34-41]. Although MEN1 tumours were previously considered to be less malignant than those occurring sporadically, it became apparent that, eventually, both primary tumours and liver metastases were the cause of significant mortality. More recently, the development of antisecretory drugs 
such as omeprazole has allowed for effective medical management of MEN1 ZES patients without the need for total gastrectomy in patients with either acute complications of peptic ulcer disease or unremitting symptoms. Because of an almost universal failure in previous surgical efforts to render MEN1 ZES patients, eugastrinemic, total gastrectomy had previously been the standard operative procedure performed in these patients. After omeprazole became available, many groups elected to treat MEN1 ZES patients with drug therapy only, avoiding an exploration unless a pancreatic tumour was imaged by $C T$ or any other localization study [42-45]. The obvious disadvantages of medical therapy in MEN1 ZES include life-long dependency on therapy with $\mathrm{H} 2$ blockers or omeprazole, the possible progression of localized malignancy to lymph nodes and liver, and the possible development of multiple gastric ECL (enterochromaffin-like tumours [46].

Our surgical approach, developed during the past 15 years, is based on the premise that MEN1 patients with neuroendocrine disease of the pancreas or duodenum can be 'cured ' of their syndrome or nonfunctional tumours, providing that the tumour has not metastasized to the liver and that the operation is extensive enough to excise all sites of disease [31-33, 38]. An aggressive surgical approach is indicated because the incidence of malignancy is similar to that in patients with sporadic islet-cell tumours, although liver metastases are less common at the time of diagnosis, perhaps because of the younger age of patients at the time of diagnosis and the frequency of duodenal primary tumours in MEN1 ZES patients $[18,40,43]$.

\section{MEN1 non-functional tumours}

Both benign and malignant neuroendocrine tumours develop in the pancreas in patients with MEN1. In our experience, the majority of patients with functional tumours have had other neuroendocrine tumours that were non-functional or secreting hormones that produce no identifiable syndrome. Additionally, some patients, estimated to represent 5-10\% of MEN1 patients, have developed neuroendocrine tumours producing symptoms entirely related to their size. local invasion or hepatic metastases. Three recent patients illustrate the management of these tumours. A 41-year-old male patient with no family history of MEN1 developed

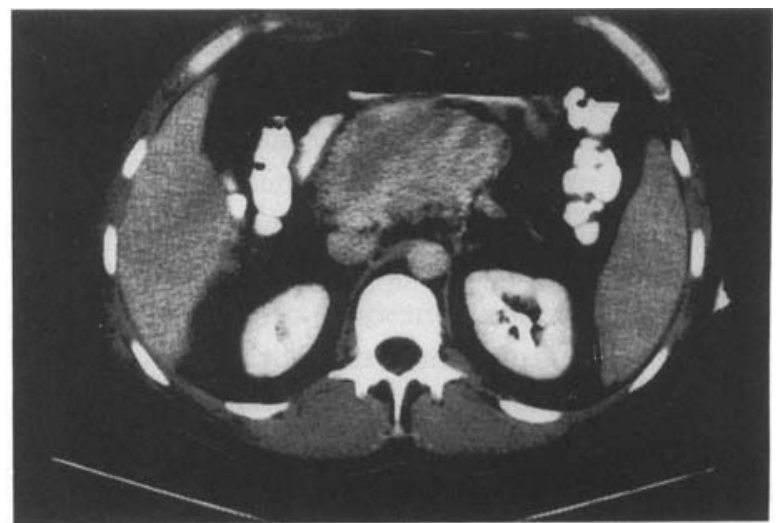

Fig. 1 CT scan of a 41-year-old MEN 1 male showing a $12-\mathrm{cm}$ tumour arising from the neck and the body of the pancreas with obstruction of the splenic and superior mesenteric veins with portal hypertension. There was no evidence of liver metastases.

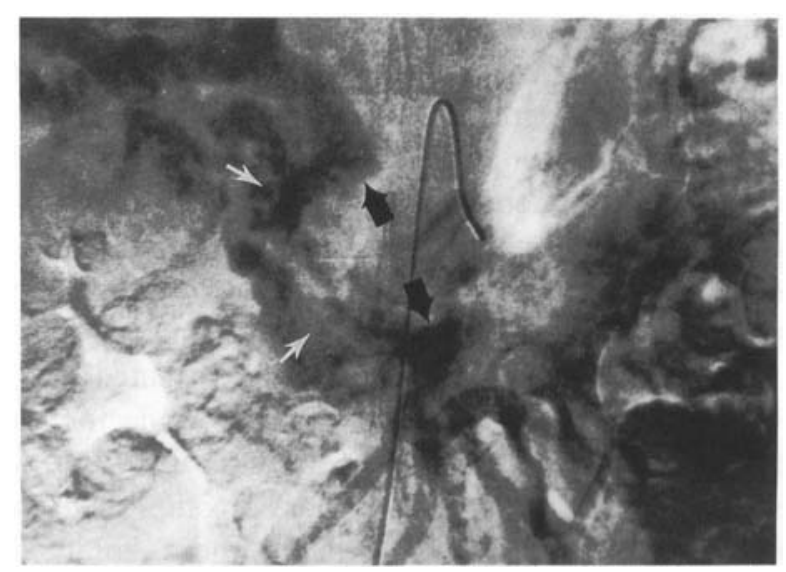

Fig. 2 Venous phase in same patlent as Fig. 1: selective visceral angiogram showing complete occlusion of the splenic and distal superior mesenteric and proximal portal veins. Note reconstruction of portal veins from large venous collaterals (white arrows) coursing through the pancreatic head. Black arrows point to proximal SMV and distal portal vein.

hyperparathyroidism at 37 years of age. Parathyroid explorations were performed elsewhere in 1987 and 1990 for what was eventually determined to be asymmetrical parathyroid hyperplasia. During the autumn of 1991, he developed crampy abdominal pain, a $9 \mathrm{~kg}$ weight loss and a palpable abdominal mass. A CT scan showed a $12-\mathrm{cm}$ mass in the pancreas in the region of its neck and body [Fig. 1]. An angiogram showed a large hypervascular mass completely occluding the superior mesenteric, splenic and proximal portal veins [Fig. 2]. Imaging showed 

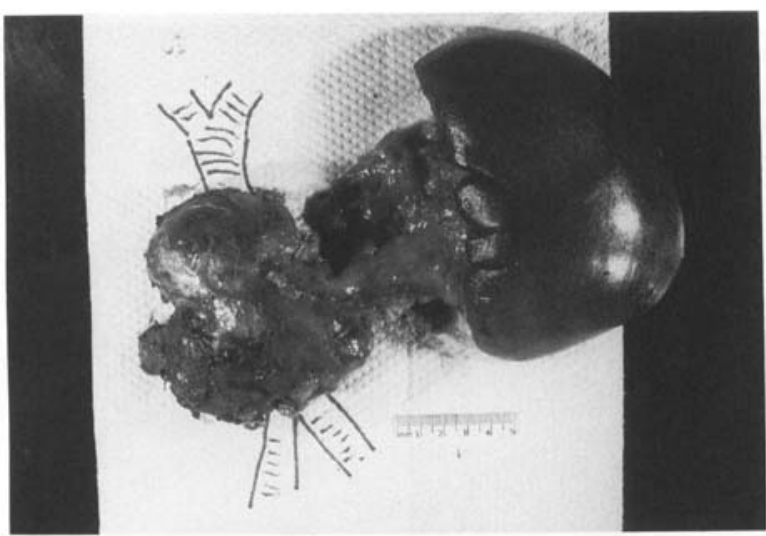

Fig. 3 The operative specimen showing a 12-cm invasive neuroendocrine tumour involving the mid-portion of the pancreas and contiguous veins. The superior mesenteric and proximal portal veins were resected and replaced with a ribbed dacron graft.

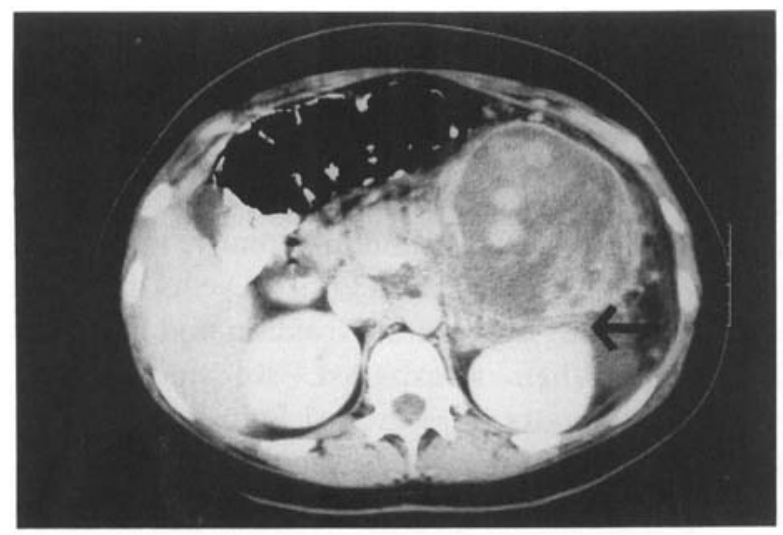

Flg. 4 CT scan in a 22-year-old female MEN1 patient who developed left upper quadrant pain 3 years after a subtotal parathyroidectomy and thymectomy. When 19 years of age. screening studies for endocrine disease of the pituitary, pancreas and adrenal glands had been negative. GI hormone levels were normal at the time of this CT except for a slightly elevated serum gastrin and a positive secretin test. CT demonstrates a 13-cm tumour involving the body and tall. The arrow points to non-Invaded plane between the tumour and the left kidney.

no liver metastases. Because of progressive pain and weight loss, an exploration was performed in January 1990. After a difficult dissection, it was possible to isolate the proximal superior mesenteric and distal portal veins for interposition of a ribbed dacron vascular graft after a distal pancreatectomy which included the enlarged tumour and invaded portal and superior mesenteric veins [Fig. 3]. Despite a complicated post-operative recovery, this patient did well for the next 3 years and is working full-time, although recently, a CT scan has shown possible

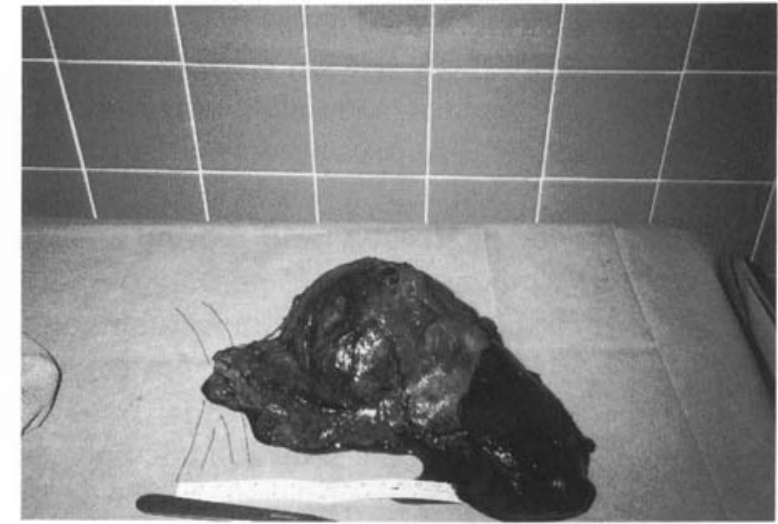

Fig. 5 The operative specimen of resected distal pancreas, tumour and spleen. A 2-mm gastrinoma was excised from the second portion of the duodenum. No histological evidence of malignancy was found in lymph nodes, and liver was negative for metastases.

local tumour recurrence and liver metastases.

A 22-year-old female with a known family history of MEN1 was found to be hypercalcemic at 19 years of age after developing a renal stone. A subtotal parathyroidectomy and cervical thymectomy were performed. and she remained asymptomatic until 3 years later, when she suffered left upper quadrant pain for several weeks following a minor fall on ice. A CT scan then demonstrated a $13-\mathrm{cm}$ mass in the distal pancreas and a normal spleen and liver [Fig. 4]. The only abnormality in her hormonal screen was a slightly elevated serum gastrin $\left(160 \mathrm{mg} \mathrm{mL}^{-1}\right)$. A secretin test was positive for gastrinoma. At exploration in March of 1993, a large tumour involving the entire body and tail was resected with the spleen [Fig. 5]. There was no evidence of local invasion, or lymph node or liver metastases. A duodenotomy was also performed and a 2-mm neuroendocrine tumour was locally excised from the distal second portion of the duodenum. Peripancreatic lymph nodes were negative for metastases. The duodenal microadenoma stained positive for gastrin, whereas the large neuroendocrine tumour, which was histologically benign. stained only for neuron specific enolase (NSE) and chromogranin. She has remained asymptomatic since the operation and has no displayed evidence of recurrence.

The third patient, a 54-year-old female, presented with recurrent hyperparathyroidism 10 years after a 
subtotal parathyroidectomy for multiple gland disease. At that time, there was no family history of MEN1, but subsequently, a daughter developed a prolactinoma and HPT. As a result, she had hormonal screening and a CT scan which demonstrated a $3-\mathrm{cm}$ tumour within the pancreatic head. During a combined operation consisting of parathyroid reexploration and abdominal exploration, the neuroendocrine tumour in the head was enucleated, and a distal pancreatectomy was performed for multiple small palpable tumours in the body and tail. She has been asymptomatic and normocalcemic for the last 2 years. None of these tumours were histologically malignant.

The results from this group of patients suggest that periodic screening of the pancreas in all proven MEN1 patients is indicated. In most patients without liver metastases, determining whether a tumour is benign or malignant cannot be made until resection. histopathological study, and in some cases, longterm follow-up.

\section{Functional islet-cell tumour syndromes}

A spectrum of islet-cell tumours syndromes have been reported in MEN1 patients, including VIP oma, glucagonoma, ectopic ACTH and somatostatinoma. Only the two most common, resulting from insulinomas and gastrinomas, will be considered in further detail.

\section{Insulinoma}

It is estimated that $10-15 \%$ of MEN 1 patients will develop insulinomas as the only functional component in their pancreatic neuroendocrine disease. Approximately $5 \%$ of all insolinomas occur in MEN1 pattents. As a general rule, sporadic insulinomas are solitary tumours, whereas insulinomas in MEN1 patients are likely to be multiple. Nevertheless. multiple tumours in MEN1 patients are not always insulinomas, and their designation as such must be proven by immunohistochemical staining $[8,18-20,31,41,44-45]$. Whenever a presumed sporadic insulinoma patient is found to have multiple islet-cell tumours, a complete evaluation for MEN1 should be undertaken. Most of these patients will eventually be proven to have the syndrome.

Five patients with symptomatic insulinomas occurring in association with the MEN1 syndrome

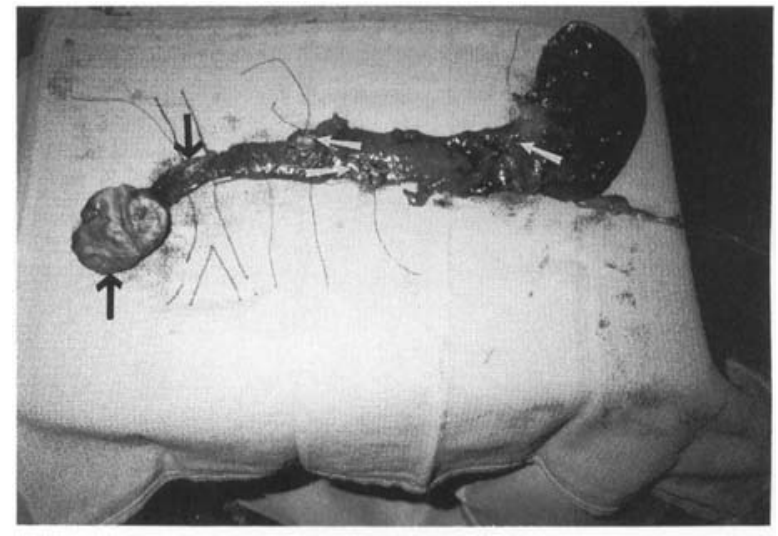

Fig. 6 Operative specimen from a 27-year old MEN1 male with a $7-\mathrm{cm}$ pedunculated malignant insulinoma, a $2-\mathrm{cm}$ benign insulinoma (black arrows) and three non-functional neuroendocrine tumours (white arrows).

were treated at the University of Michigan over the past 20 years. One additional patient, operated upon elsewhere, underwent an enucleation of an insulinoma located in the tail of the pancreas at the age of 9 years. At that time, there was no known family history of MEN1, and apparently, this was a solitary tumour. She did well until 35 years of age, at which time both hyperparathyroidism and ZES were diagnosed. When re-explored. six macroneuroendocrine tumours were excised from the pancreas and seven microgastrinomas from the duodenum. At least two or more insulinomas were excised in each of the other five patients. One patient, a 27-year-old male with a large $(7-\mathrm{cm})$ predunculated insulinoma arising from the neck of the pancreas was also found to have an additional insulinoma, as well as three other nonfunctioning neuroendocrine tumours [Fig. 6]. The largest insulinoma was histologically malignant and one contiguous lymph node contained a metastatic neuroendocrine tumour which stained positively for insulin. Two patients developed insulinomas within 2 and 3 years, respectively, after the surgical treatment of ZES, successfully treated by excision of duodenal gastrinomas. Neither patient had undergone a distal pancreatectomy at the time of their first operation. Multiple insulinomas were found in the body and tail in each patient at reoperation. All six patients are euglycemic at the present time with follow-ups ranging from 2 to 16 years.

Although we relied upon percutaneous transhepatic selective venous sampling of insulin for 
Fig. 7 Algorithm of a management plan currently employed in MEN 1 patients with hyperinsulinism. Endoscopic ultrasound is the only localization study used primarily to evaluate the head and uncinate process of the pancreas.

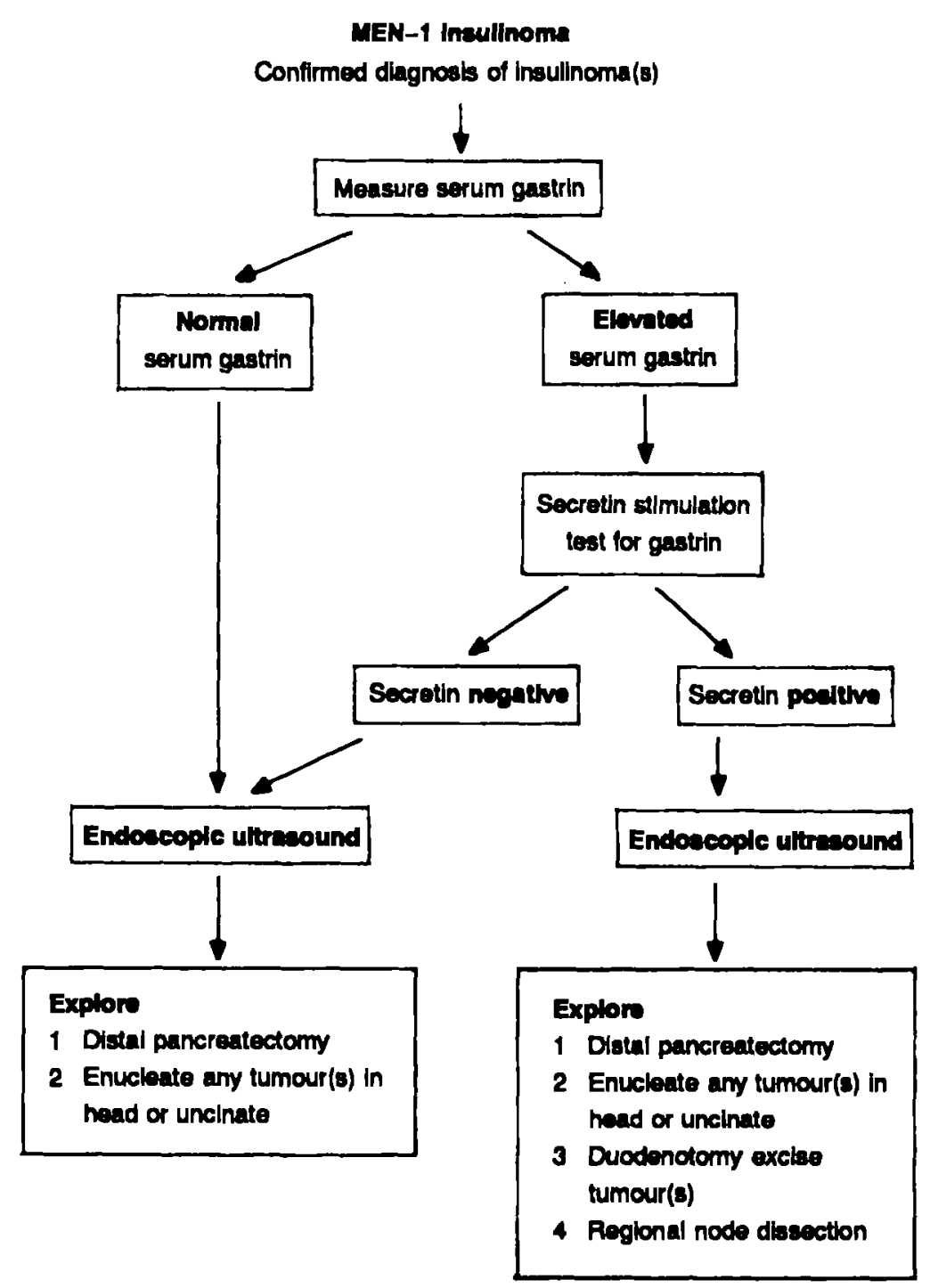

localization of insulinomas for more than a decade. we no longer consider that study necessary [19]. The only localization we recommend in MEN1 patients is endoscopic ultrasonography of the head and uncinate process of the pancreas. Our current management algorithm is shown in Fig. 7. In addition to a confirmed biochemical diagnosis of organic hyperinsulinism, a serum gastrin level is obtained to rule out concomitant preclinical gastrinoma.

The operative procedure we perform is a distal pancreatectomy to the level of the superior mesenteric vein. and enucleation of any neuroendocrine tumours in the head or uncinate process. A duodenotomy is not performed unless the pre-operative serum gastrin levels are elevated and a secretin test is positive. Extrapancreatic insulinomas are extremely rare and have not been reported in MEN1 patients. In our experience, neuroendocrine duodenal tumours in both MEN1 and sporadic patlents have rarely shown any cells staining positive for insulin. A lymphatic dissection is performed only in those pattents with concomitant duodenal gastrinomas or in the rare MEN1 patient with a malignant insulinoma.

\section{Gastrinomas}

The surgical treatment of ZES in MEN1 patients is predicated on the facts that all gastrinomas, whether in the duodenum or the pancreas, have malignant 


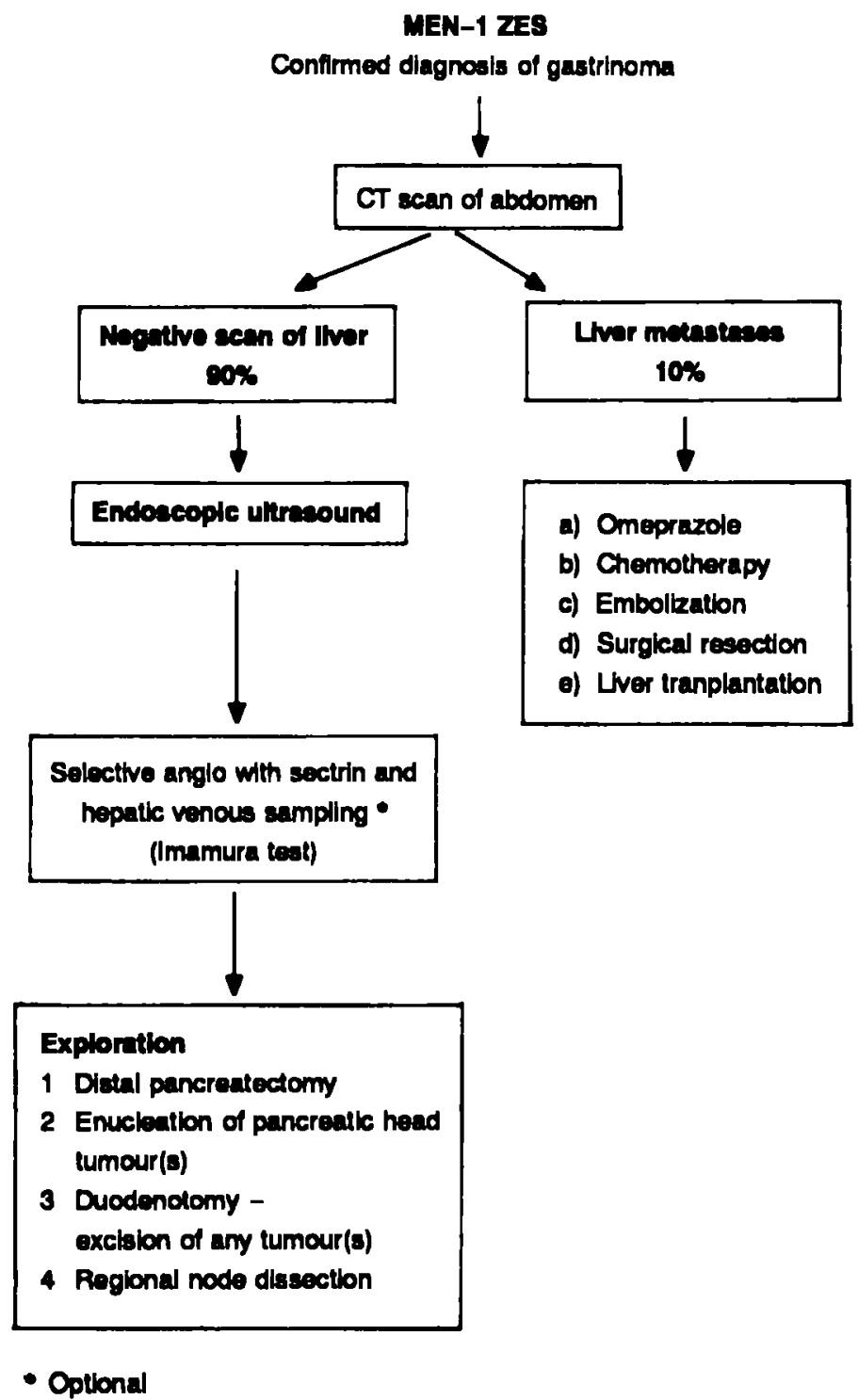

Fig. 8 Algortthm of a management plan in MEN1 ZES patlents. Ejther CT or MRI is used to evaluate the llver for metastases and the adrenal glands for possible cortical tumours. Endoscople ultrasound is used to evaluate the head and uncinate process. Duodenal microadenomas present in the majority of these patients are usually too small to be detected by any localization technique. potential or are already malignant, and that surgical treatment can be successful in patients without liver metastases, providing the operative procedure performed is appropriate. All patients without liver metastases should be explored with the intent to 'cure' the disease, short of a Whipple procedure or total pancreatectomy. The following principles are guidelines in all explorations for ZES in MEN1 patients: (1) A duodenotomy is performed and the wall is circumferentially palpated from the pylorus through the third portion. Any small (less than $0.5 \mathrm{~cm}$ ) gastrinomas are locally excised. Larger gastrinomas are excised with a full thickness margin of duodenal wall. (2) All peripancreatic lymph nodes are excised, including those along the common bile duct and within the porta hepatis medially to the celiac axis. (3) After full mobilization of the head and uncinate, any palpable or ultrasonically identified tumour is enucleated. (4) A distal pancreatectomy, including the neck, body and tail, preserving the spleen when feasible, is routinely performed. The majority of MEN1 patients have palpable neuroendocrine tumours within the body or tail. The only pre-operative localization studies we now utilize are a CT or MRI scan to rule out liver metastases and to evaluate the adrenal glands. Endoscopic ultrasonography is used to evaluate the head and uncinate process for possible neuro- 
Table 1 Location of Immunohistochemically proven gastrinomas in 17 patients with MEN1 ZES

\begin{tabular}{lr}
\hline Location & \multicolumn{1}{c}{ No. (\%) } \\
\hline Gastrinoma(s) in duodenum & $13(76 \%)$ \\
Gastrinomas(s) in duodenum and pancreas & $6(35 \%)$ \\
Multiple gastrinomas in duodenum & $8(47 \%)$ \\
Gastrinoma(s) in pancreas & $10(59 \%)$ \\
Gastrinomas(s) only in pancreas & $4(24 \%)$ \\
\hline
\end{tabular}

endocrine tumours too small to be palpated during exploration. An algorithm of our current management scheme is shown in Fig. 8.

During the 15-year period from 1978 to 1993,17 MEN1 patients without liver metastases underwent pancreatic exploration with intent to 'cure' their biochemically proven ZES. Eleven patients were female and six were male. Their mean age was 36.7 years at the time of exploration. Twelve patients had previously undergone a parathyroidectomy, whereas five with a synchronous diagnosis of HPT and ZES were treated with a combined operation.

Gastrinoma(s) were found in the duodenum of $76 \%$ and in both pancreas and duodenum of $35 \%$ (Table 1). A minimum of $46 \%$ with duodenal gastrinomas were proven to be malignant, as determined by lymph node metastases. Gaastrinoma(s) were only found in the pancreas in $24 \%$, although $59 \%$ had pancreatic gastrinomas in the entire group. None of the pancreatic gastrinomas have proven to be malignant, as determined by local invasion. lymph node or liver metastases. Other neuroendocrine tumours were found in the body or tail of the pancreas at the initial exploration in $82 \%$. Additional neuroendocrine tumours developed which were proven at a subsequent exploration in the remaining three patients after periods ranging from 2 to 8 years. Thus, $100 \%$ of these patients had or developed neuroendocrine tumours of the body or tail.

Thirteen out of 17 patients (76\%) had a distal pancreatectomy as a component of their initial operation. Of the four with a remaining body and tall, one with three duodenal and two pancreatic head gastrinomas had lower but persistent hypergastrinemia. At a second exploration, 2 months later, a 1.5-cm gastrinoma was found and excised from the distal pancreatic tail. Another patient had normal basal gastrin levels but a positive secretin study for 8 years. At re-exploration, a $1.5-\mathrm{cm}$ gastrinoma was found and excised from the pan- creatic neck. Both patients have had normal gastrin levels and negative secretin tests for periods of 4 and 5 years.

The other two patients both became eugastrinemic after excision of gastrinomas from the duodenum. but developed organic hyperinsulinism after 2 and 3 year intervals, respectively. At re-exploration, multiple insulinomas were found in the distal pancreas in both patients. These patients were successfully treated by distal pancreatectomy.

After follow-up periods ranging from one to 16 years. 11 out of $17(65 \%)$ patients have normal basal gastrin levels, are asymptomatic, and require no drug therapy for diarrhoea or peptic ulcer symptoms. Five of these patients have had one or more negative secretin-stimulated studies (29\%). The six patients (35\%) currently with increased basal gastrin levels have all had a decrease in gastrin levels, a decrease in symptoms and a decrease in drug requirements for variable periods after operation, although none became eugastrinemic. Because none of these patients have liver metastases, they are considered potential candidates for a re-exploration. One of these patients has recently undergone endoscopic ultrasonography and two $0.5-\mathrm{cm}$ neuroendocrine tumours were identified in the remaining pancreatic head.

\section{Discussion}

Neuroendocrine tumours of the pancreas develop in $50 \%$ of patients with MEN1 and may cause significant morbidity or mortality $[5,18,41,45,46]$. Their treatment is surgical when detected by imaging. biochemical screening or biochemical confirmation of a hormonal syndrome. The objective in all patients is to detect and excise the tumour(s) before their malignant potential has been confirmed by hepatic metastases. In patients with functional syndromes caused by hypersecretion of a specific hormone, surgical treatment may be life saving (glucagonoma, VIPoma or insulinoma), or eliminate the need for expensive daily drug therapy (gastrinoma). These objects need to be re-emphasized in MEN1 patients with ZES because many groups have excluded these patients for operation based on historical failures caused by previous inadequate knowledge of tumour pathophysiology.

Our current operation has evolved during a 15year period, and is based on an improved under- 
standing of the extent and locations of gastrinomas in MEN1 [7, 11,30-33, 38]. The fact that some patients become eugastrinemic yet have positive secretin stimulation tests suggests that either a very small duodenal or pancreatic head gastrinoma, or micrometastases in a lymph node was overlooked at exploration. Initially, it was assumed that all of these patients would develop symptomatic recurrences, but surprisingly, that has not been the case [33]. One patient in this category has been symptom free without drug therapy for 16 years, and two others had similar courses for 8 and 9 years, respectively, before developing recurrent hyperparathyroidism and then elevated basal serum gastrin levels. In both cases, successful parathyroid re-operations resulted in eugastrinemia, which has been maintained for 4 and 6 years, respectively [47]. Whether MEN1 patients with hypergastrinemia have a greater propensity to have concomitant neuroendocrine tumours in the pancreas is not known. The fact that $100 \%$ of our patients had or developed neuroendocrine tumours in the body or tail of the pancreas is the basis for our recommendation to include a distal pancreatectomy as a routine step in the MEN1 ZES operation. An alternative approach, which has been advocated by several authors, is to perform a pancreaticoduodectomy resection which would eliminate any neuroendocrine tumours in the duodenum, head, uncinate and peripancreatic lymph nodes $[16,37]$. Any tumour in the remaining pancreatic body and tail could be enucleated. It is not unreasonable to assume that an even higher cure rate of the ZES could be achieved with this procedure. Whether the potential added morbidity and possible mortality associated with this operation is justifiable remains to be answered. In those patients who are not cured after the procedure, we perform a 'second look' procedure, which can be performed after a year or so with the reasonable assumption that a previously occult tumour may have grown to detectable size. We are currently considering re-exploration in several patients who have had a rising gastrin level after a previous operation failed to return the serum gastrin level to the normal range. In one of these patients, two small neuroendocrine tumours have been identified in the remaining pancreatic head by endoscopic ultrasound. Another approach would be to excise all duodenal tumours and to enculeate any pancreatic tumours rather than resect the distal pancreas. Failures after this procedure would still be
Table 2 The occurrence of malignancy in MEN1 ZES patients

\begin{tabular}{ll}
\hline Occurrence & No. (\%) \\
\hline Malignant duodenal gastrinoma(s) & $6(46 \%)$ \\
Malignant pancreatic gastrinoma(s) & $0(0 \%)$ \\
Malignant E pancreatic tumour & $1(6 \%)$ \\
Liver metastases including follow-up & $0(0 \%)$ \\
(range 1-15 years) & \\
\hline
\end{tabular}

amenable to a pancreaticoduodenectomy without resorting to a total pancreatectomy. In patients without liver metastases, a total pancreatectomy. including any involved lymph nodes, would cure the MEN1 patient of pancreatic and duodenal neuroendocrine disease. Considering the potential longterm side effects of this procedure and the relative indolence of most neuroendocrine malignancies of MEN1, the benefits gained would be likely to overcome by the subsequent morbidity in all but the rare MEN1 families with proven highly aggressive pancreatic malignancies [48].

Although our series is small, several observations can be emphasized. The first is that the majority of the MEN1 ZES patients can be rendered eugastrinemic if diagnosed and explored before liver metastases have developed (Table 2). The second is that excision of tumours and lymph node metastases when present may prevent the subsequent progression to liver metastases. Although our longest follow-up in this series is only 16 years, no patient has developed liver metastases. These initial results encourage us to recommend pancreatic duodenal exploration in all MEN1 ZES patients without liver metastases. There is increasing evidence that, after a procedure appropriate for the neuroendocrine disease present, most patients will obtain significant longterm benefits. To do nothing but observe and treat with drug therapy until a tumour is obvious may eliminate the potential opportunity to cure many of these patients.

\section{References}

1 Allo M. Thompson NW. Hyperparathyroidism as a part of the MEN I Syndrome. In: Kaplan EL, ed. Surgery of the Thyroid and Parathyroid Glands. Clinical Surgery International. Churchill Luvingston. 1983: 177-92.

2 Block MA. Famllial hyperparathyroldism and hyperparathyroldism assoclated with multiple endocrine neoplasla syndrome. In: Cady B. Rossi RL, eds. Surgery of the Thyrold and Parathyroid Glands, 3rd edn. Philadelphia, PA: W. B. Saunders \& Co., 1991. 
3 Lamers CB. Froeling PG. Clinical significance of hyperparathyroidism in familial multiple endocrine adenomatosis type I (MEA I). Am J Med 1979: 66: 422-4.

4 Malmaeus J. Benson L. Johansson H. Ljunghall S. Rastad J. Akerstrom G. et al. Parathyrold surgery in the multiple endocrine neoplasia type I syndrome: Choice of surgical procedure. World J Surg 1986: 10: 668-72.

5 Shepherd JJ. The natural history of multiple endocrine neoplasia type I. Arch Surg 1991: 126: 935-46.

6 Donow C. Pipeleers-Marichal M, Schroder S. Surgical pathology of gastrinoma: site, size, multicentricity, association with multiple neoplasia Type I and malignancy. Cancer 1991; 68: 1329-34.

7 Thompson NW, Lloyd RB, Nishiyama RH. Vinik AI. Strodel WE, Allo MD, et al. MEN-I pancreas: A histological and immunohistological study. World I Surg 1984; 8: 561-74.

8 Kloppel G, Willemer S. Stamm B, Hack! WH, Heitz PU. Pancreatic lesions and hormonal profile of pancreatic tumors in multiple endocrine neoplasia type I: An immunocytochemical study of nine patients. Cancer 1986; 57: 1824-32.

9 MacGillivay DC, Rushin JM. Zeiger MA. Shakir KMM. The significance of gastrinomas found in peripancreatic lymph nodes. Surgery 1991: 109: 556-62.

10 Pipeleers-Marichal M, Somers G. Willems G, Foulis A, Imrie C, Bishop AE, et al. Gastrinomas in the duodenums of patients with multiple endocrine neoplasia type I and the ZollingerEllison syndrome. $N$ Engl $J$ Med 1990; 322: 723-7.

11 Vinik AI, Moattari AR, Cho K. Thompson NW. Transhepatic portal vein catheterization for localization of sporadic and MEN gastrinomas: A ten-year experience. Surgery 1990; 107: 246-55.

12 Imamura M. Takahashi K, Adachi H, Minematsu S, Shimada Y. Naito M. Suzuki T. et al. Usefullness of selective arterial secretin injection test for localization of gastrinoma in the Zollinger-Ellison Syndrome. Ann Surg 1987: 205: 230-6.

13 Delcore R. Jr. Cheung LY, Friesen SR. Characteristics of duodenal wall gastrinomas. Am J Surg 1990; 160: 621-4.

14 Thom AK, Norton JA. Axiotis CA. Jensen RT. Location, incidence and malignant potential of duodenal gastrinomas. Surg 1991; 110: 1086-93.

15 Bornman PC, Marks IN, Mee AS. Price S. Favorable response to conservative surgery for extra-pancreatic gastrinoma with lymph nodes metastases. Brit J Surg 1987: 74: 198-201.

16 Imamura M. Kanda M. Takahashi K. Shimada Y, Miyahara T. Wagata T. et al. Clinicopathological characteristics of duodenal microgastrinomas. World J Surg 1992; 16: 703-10.

17 Sugg SL. Norton JA, Fraker DL, Metz DC, Pisegna JR, Fishbeyne V. et al. A prospective study of intraoperative methods to diagnose and resect duodenal gastrinomas. Ann Surg 1993: 218: 138-44.

18 Shepherd JJ, Challis DR, Davies PF, McArdle JP, Teh BT, Wilkinson $S$, et al. Multiple endocrine neoplasia, type I: gastrinomas. pancreatic neoplasms, microcarcinoids, the Zollinger-Ellison Syndrome. lymph nodes and hepatic metastases. Arch Surgery 1993: 128: 1133-42.

19 Pasieka JL, Mcleod MK. Thompson NW. Burney RE. Surgical approach to insulinomas: Assessing the need for preoperative localization. Arch Surg 1992: 127: 442-7.

20 Service FJ, McMahon MM. O'Brien PC. Ballard DJ. Functioning Insulinoma-incidence recurrence and long-term survival of patients: A 60-year study. Mayo Clin Proc 1991 : 66: 711-19.
21 Akerstrom G. Malmaes J. Bergstrom R. Surgical anatomy of human parathyroid glands. Surgery $1984: 95: 14-21$.

22 Block MA. Frame B. Jackson CF. The efficacy of subtotal parathyroidectomy for primary hyperparathyroldism due to multiple gland involvement. Surg Gynecol Obstet 1978: 147: 1-5.

23 Lght GS. Hensley MI. Management of famillal hyperparathyroidtsm. Prog Surg 1986: 18: 106-16.

24 Prnz RA, Gamvros OI, Sellu D, Lynn JA. Subtotal parathyroidectomy for primary chief cell hyperplasia of the multiple endocrine neoplasia type I syndrome. Ann Surg 1981; 193: 26-9.

25 Rtzzoll R, Green J, III, Marx SJ. Primary hyperparathyroidism in familial multiple endocrine neoplasia type I. Long-term follow-up of serum calcium levels after parathyroidectomy. Am J Med 1985; 78: 467-74.

26 Thompson NW. The techniques of initial parathyroid explorative and reoperative parathyroidectomy. In: Thompson NW, Vinik AI, eds. Endocrine Surgery Update. New York, NY: Grune \& Stratton, 1983: 365-83.

27 Wells SA, Jr, Farndon JR, Dale JK, Leight GS, Dilley WG. Longterm evaluation of patients with primary parathyroid hyperplasia managed by total parathyroidectomy and heterotopic autotransplantation. Ann Surg 1980; 192: 451-8.

28 van Heerden JA, Kent RB. III, Stzemore GW, Grant CS, ReMine WH, Kent RB. Primary hyperparathyroidism in patients with multiple endocrine neoplasia syndrome. Surgical experience. Arch Surg 1983; 118 : 533-6.

29 Thompson NW. Eckhauser FE. Harness JK. Anatomy of primary hyperparathyroidism. Surgery 1982; 92: 814-22.

30 Thompson NW, Vinik AI, Eckhauser FE. Microgastrinomas of the duodenum. A cause of falled operations for the ZollingerEllison syndrome. Ann Surg 1989; 209: 396-404.

31 Thompson NW. The surgical treatment of the Zollinger-Ellison Syndrome in sporadic and MEN I Syndrome. Acta Chir Austriaca $1992 ; 24: 82-7$.

32 Thompson NW. The surgical treatment of the endocrine pancreas and Zollinger-Ellison syndrome in the MEN-I Syndrome. Henry Ford Hospital Med Journal 1992; 40: 195-198.

33 Thompson NW. Bondeson AG, Bondeson L. Vinik AI. The surgical treatment of gastrinoma in MEN-I syndrome patients. Surgery 1989; 106: 1081-6.

34 Delcore R. Herumreck AS. Friesen SR. Selective surgical management of correctable hypergastrinemia. Surgery 1989 : 106: 1094-102.

35 Delcore R. Friesen SR. Zollinger-Ellison Syndrome. A new look at regression of gastrinomas. Arch Surg 1991 : 126: 556-8.

36 Friesen SR. Are ' aberrant nodal gastrinomas ' pathogenetically similar to 'lateral aberrant thyrold' nodules? Surgery 1990 ; 107: 236-8.

37 Delcore R, Friesen SR. The role of pancreatoduodenectomy of primary duodenal wall gastrinomas in patients with the Zollinger-Ellison Syndrome. Surgery 1992: 112: 1-8.

38 Thompson NW, Pasieka J, Fukuuchl A. Duodenal gastrinomas. duodenotomy and duodenal exploration in the surgical management of Zollinger-Ellison Syndrome. World $J$ Surg $1993 ; 17: 455-62$.

39 PJpeleers-Marichal M. Donow C. Heltz PU. Kloppel G. Pathologic aspects of gastrinomas in patients with Zollinger-Ellison Syndrome with and without multiple endocrine neoplasia Type I. World I Surgery 1993; 17: 481-8.

40 Cheruer JA. Sawyers JL. Benefit of resection of metastatic 
gastrinoma in Multiple Endocrine Neoplasia Type I. Gastroenterology 1992; 102: 1049-53.

41 Akerstrom G. Johansson H. Grama D. Surgical treatment of endocrine pancreatic lesions in MEN I. ACTA Oncologica 1991: 30: 541-5.

42 van Heerden JA, Smith SL, Miller L. Management of the Zollinger-Ellison Syndrome in patients with multiple endocrine neoplasia type I. Surgery 1986; 100: 971-6.

43 Norton JA. Doppman JL, Jensen RT. Curative resection in Zollinger-Ellison Syndrome: Results of a 10 year prospective study. Ann Surg 1992; $215: 8-18$.

44 Mignon M, Ruszniewski P. Podevin P, et al. Current approach to the management of gastrinoma and insulinoma in adults with multiple endocrine neoplasia I. World J Surgery 1993: 17: 489-97.

45 Sheppard BC, Norton JA. Doppman JL, et al. Management of islet-cell tumors in patients with multiple endocrine neoplasia : a prospective study. Surgery 1989; 106: 1108-18.
46 Solcia E. Capella C. Flocca R. Rindi G. Rosal J. Gastric argyrophil carcinoidosis in pattents with Zollinger-Ellison Syndrome due to Type I multiple Endocrine Neoplasia. Am I Surg Pathol 1990: 14: 503-13.

47 Norton JA. Cornelius MJ. Doppman JL, Maton PN, Gardner JD, Jensen RT. Effect of parathyroidectomy in patients with hyperparathyroidism. Zollinger-Ellison syndrome and multiple endocrine neoplasia type I: A prospective study. Surgery 1987: 102: 958-66.

48 Tisell LE, Ahlman H. Jansson S. Grimelius L. Total pancreatectomy in the MEN-I syndrome. $B r /$ Surg 1988: 75: $154-7$.

Received 18 October 1994 : accepted 15 May 1995.

Correspondence: Norman W. Thompson MD. University of Michigan. Department of Surgery. 1500 E Medical Center Drive. Ann Arbor, MI 48109. USA. 